\title{
Searching and Rescuing Victims in Emergency: A Comprehensive Survey
}

\author{
Huibo $\mathrm{Bi}^{\mathrm{a}}$ and Erol Gelenbe ${ }^{\mathrm{b}, 1}$ \\ ${ }^{a}$ Beijing Key Laboratory of Traffic Engineering, College of Metropolitan \\ Transportation, Beijing University of Technology, Beijing 100124, China \\ ${ }^{\mathrm{b}}$ Institute of Theoretical \& Applied Informatics, Polish Academy of Sciences \\ (IITIS-PAN), Battycka 5, 44-100 Gliwice, Poland
}

\begin{abstract}
The rapid progress in urban construction and informatization, especially the recent ubiquity of portable devices and intelligent infrastructures, has rapidly aggravated the concentration of pedestrians in built environments. These smart environments, which have been driven close to their limitation in daily operations, can be vulnerable during emergency situations and amplify the losses in lives and property. However, like every coin has two sides, the extra resources brought by smart equipments enlarge the optimisation space of an emergency search and rescue process and provide opportunities for new system frameworks and algorithms. In this paper, we review the current state and opportunities of the pedestrian search and rescue problem. key factors including system design, pedestrian dynamic modelling, and algorithm development are reviewed and summarised.
\end{abstract}

Keywords. Emergency management, Emergency Search and Rescue, Systemic Review

\section{Introduction}

As one of the most active research fields, emergency search and rescue planning, which features the widening gap between instant vast demands and restricted resources, has always been the favourite test field for cutting-edge technologies in computation, communication, and control. The basic aim of emergency search and rescue planning is to rescue victims out of dangerous areas while minimising the safety cost. However, this problem can be NP-hard due to the highly dynamic environments and complex interactive dynamics among pedestrian flows and information flows. Owing to its multidisciplinary nature, efforts have been made by scientists and sociologists in many domains to investigate this problem. In this paper, we focus on the computer-aided solutions of the emergency search and rescue planning problem.

The human history has seen a transformation in emergency management from a unorganized, reactive manner to an organised, proactive manner. Nowadays, an overall emergency management process can be divided into four aspects, emergency prevention, emergency preparedness, emergency response and emergency recovery. Emergency

\footnotetext{
${ }^{1}$ Corresponding Author: Erol Gelenbe, Institute of Theoretical \& Applied Informatics, Polish Academy of Sciences (IITIS-PAN); E-mail: gelenbe.erol@gmail.com.
} 
search and rescue planning is the key research direction of the emergency response section. Originating from maritime search and rescue operations, search and rescue planning in emergency situations has motivated considerable research over the last several decades owing to the unfortunate increasing threat of both manmade and natural disasters. During a disaster-related emergency evacuation, evacuees may become immobilised and incapacitated due to injuries or obstacle contact. Therefore, to reduce the fatalities, various emergency management systems have been proposed to detect the location of incapacitated evacuees and dispatch rescuers to perform rescue operations. The main challenges of a rescue operation are threefold. The first challenge is how to efficiently search and locate injured evacuees or other objects in unknown environments, especially on how to coordinate the activities among various rescuers. The second is to design an appropriate rescuer assignment algorithm to allocate rescuers to injured evacuees in a real time and computationally efficient fashion under the highly dynamic hazardous environment. This is actually a NP-hard assignment problem [1], which aims to minimise the overall potential cost for the rescue operation. The third challenge is to search desired paths for rescuers and victims to fulfil their specific requirements, which is difficult since the "quality" of a path is affected by the spreading of the hazard, the dynamic congestion level, the movements and behaviours of evacuees and other rescuers on the path. In the following sections, we will summarise various systems and algorithms that have been proposed to meet the aforementioned challenges. A tree diagram of the reviewed research topics can be seen in Figure 1.

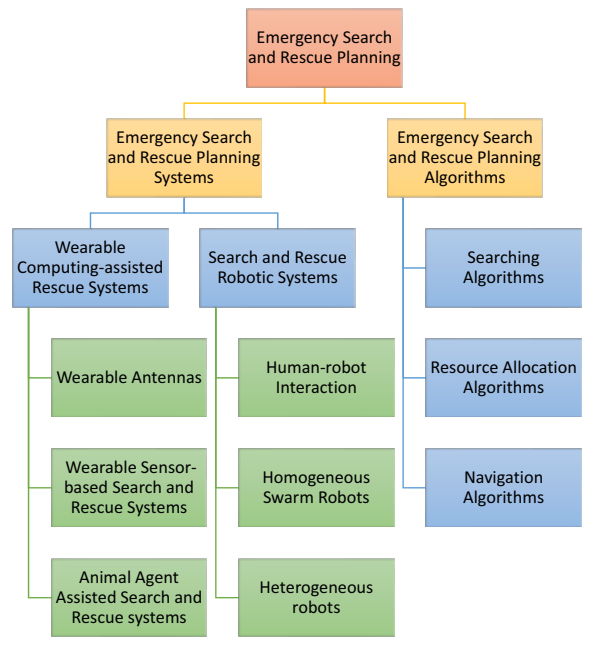

Figure 1. A tree diagram explaining the structure of the review.

\section{Emergency Search and Rescue Planning Systems}

Based on the facilities used, emergency search and rescue planning systems can be divided into two categories, wearable computing-assisted rescue systems and search \& rescue robotic systems. Wearable computing-assisted rescue systems concentrate on provid- 
ing various enhancements for emergency personnel with the aid of wearable devices to increase the efficiency of rescue operations and improve the safety of emergency responders. On the other hand, disasters that create harsh environments with extreme temperature, toxic substances or various obstacles have exposed the unsafety and inefficiency of the human-centered search and rescue planning systems. These limitations have therefore inspired the development of the robot-centered search and rescue planning systems, which employ various mobile robots to conduct rescue operations.

\subsection{Wearable Computing-assisted Rescue Systems}

With the rising interest in body-centric wireless communications, which has also been standardised as a part of the fourth generation mobile communication systems (4G), considerable research has been dedicated to develop low cost, light-weight wearable antennas to maintain and improve emergency communications. The work in [2] implements an electrically-small wearable antenna, which is integrated into the sleeve of a jacket, to monitor the positions of emergency rescuers. The research in [3] presents an emergency rescue navigation system to direct firemen to "key corridors" to eliminate fire and congestion caused by evacuees or obstacles generated from hazard with the aid of personal digital assistants (PDAs) carried by firefighters. As an effort to decrease the likelihood of emergency events from the "prevention" aspect, the study in [4] presents a wearable personal healthcare and emergency aid system, namely "WAITER", to monitor the health status of users with wearable vital signal sensors and alert the remote healthcare center when an emergency occurs.

Owing to the superior performance of animal agents during search and rescue operations in terms of mobility, energy utilisation efficiency, sensory acuity and intrinsic cognitive capacity, much effort has been dedicated to animal agent assisted search and rescue systems. The work in [5] employs cyber-enhanced working dogs to locate and reach survivors trapped under rubble in the aftermath of large-scale disasters; this system contains three components: a smart harness worn by a working dog to monitor the surrounding environment and the canine, a remote computer carried by the handler to analyse and control the behaviours of the canine, and mobile base stations such as unmanned vehicles to maintain the communication between the working dog and the handler.

\subsection{Search and Rescue Robotic Systems}

Since the human access to the victims in the aftermath of a disaster such as an earthquake or radioactive leakage can be time consuming and may induce further casualties, the use of robots that can be released rapidly to locate and rescue victims has drawn considerable attention in recent decades, and has gradually become a research domain after the Great Hanshin earthquake and the Oklahoma City bombing in 1995. Nowadays, research on "search and rescue robotic systems" has evolved as a major branch of "search and rescue", especially for urban scale structural collapse environments. According to the different operating environments, search and rescue robots can be divided into autonomous underwater vehicles (AUVs), and urban search and rescue (USAR) vehicles, which contain various types of unmanned ground vehicles (UGVs) and unmanned aerial vehicles (UAVs). This literature review focuses on the research of USAR robotic systems, and the research on AUVs is excluded due to its distinctive emphases caused by the turbid underwater environment. 
Due to the limitations on the mobility (tracks, wheels or combination of both) and intelligence, most of the current search and rescue robot systems are not advanced enough for fully autonomous operations. Hence, a research direction is to investigate the humanrobot interaction during search and rescue operations. The first known use of USAR robots for an actual unstaged rescue mission occurs during the World Trade Center collapse, when various models of tele-operated robots from industry, military and academia were invited to assist the rescue operation.

One of the major challenges that teleoperated or semi-autonomous rescue robots face today is the high human-to-robot ratio (The human-to-robot ratio denotes the number of people needed to operate a robot), which reaches $2: 1$ or even $3: 1$ in the current state of practice. The high human-to-robot ratio restricts the scale of rescue robots to be deployed in the devastated region since too many operators can significantly increase the logistic burden and the training cost. One feasible solution to this problem is the use of homogeneous swarm robots, which are easier to control and understand. For instance, the work in [6] employs a team of homogeneous robots to search and explore several disaster sites; because of the possible environmental changes and robot failures in disaster sites, the robots are required to continuously redistribute to fulfill the desired population fraction of robots at each site. Inspired by the self-organised behavior of social insects, the study in [7] proposes a stochastic task allocation strategy to assign a swarm of homogeneous robots to various tasks during an search and rescue operation without inter-robot communications.

Owing to the diverse and complex tasks in rescue operations, most of the recently proposed USAR robot systems consist of heterogeneous robots with different capabilities instead of homogeneous robots. For instance, the study in [8] proposes a robot group which consists of three types of robots to rescue victims during nuclear plant accidents. The work in [9] presents a USAR robot system which contains aerial robots and a land robot to detect the potential survivors in high-destruction locations after an earthquake.

In addition, to verify the effectiveness of the proposed robotic systems, various real and simulated scenarios have been designed in the last two decades. The work in [10] designs a test arena with collapsed structures to evaluate the agility, planning or mapping algorithms and sensing ability of rescue robots. To mimic large scale disasters, a proving ground namely "Disaster City" with a size of $210,000 \mathrm{~m}^{2}$ has been built by the US Federal Emergency Agency (FEMA) to conduct rescue drills for robots [11].

\section{Emergency Search and Rescue Planning Algorithms}

The state-of-the-art emergency search and rescue planning studies are originated and derived from research in maritime search and rescue activities, which has been a significant research topic since 1970s. Related algorithms in emergency search and rescue planning are commonly under the assumption that multiple utilities (rescuers, robots, etc.) are involved. The reason behind this is twofold. First, time efficiency is the dominant factor for the success of a search and rescue operation, and multi-agent based search and rescue operations are obviously more efficient than single-agent based search and rescue operations. Second, multi-agent systems are generally more cost-efficient and feasible than a single agent with all the capabilities [12]. In recent decades, related algorithms have aroused a new interest in not only searching victims in hazardous environments, 
but also planetary exploration and de-mining [13]. The philosophy behind team-based search and rescue is to convert a complex problem into simpler sub-problems that are more efficient to solve. These algorithms are be classified into three types: first, searching algorithms, which are dedicated to search and locate injured evacuees in unknown environments, specifically concentrating on coordinating the search hehaviours of rescuers to produce "swarm intelligence"; second, resource allocation algorithms, which aim to assign rescuers to injured evacuees in a desired manner when the locations of evacuees are revealed; third, navigation algorithms, which focus on discovering appropriate paths for rescuers when the locations of evacuees are known. In this section, the third category is excluded since all the on-line emergency navigation algorithms are applicable to this case, and the detailed review can be found in [14].

\subsection{Searching Algorithms in Emergency Search and Rescue Planning}

The work in [15] proposes a particle swarm optimisation (PSO) based multi-robot system to find targets by gradually optimising the pre-defined goal function; each robot is modeled as a particle whose velocity and position is determined by the neighbouring particles and the previous best position of this particle. Similarly, the study in [16] designs a PSO based multi-robot system to search targets and avoid obstacles in an unknown environment; specially, a relative coordinate system is used to avoid the dependence of the precise global location of robots. By employing a bio-inspired random search behaviour called lévy flight, the work in [17] presents an efficient multi-robot searching algorithm to search targets in an unknown environment.

\subsection{Resource Allocation Algorithms in Emergency Search and Rescue Planning}

The work in [1] presents a resource allocation algorithm on the basis of the Random Neural Network (RNN) with synchronised interactions [18] to assign rescuers to trapped victims in a fire-affected building; to optimise this task assignment process, several effect factors such as the cost of assigning a rescuer to a victim (e.g. the distance between them), the probability that a rescuer fails to save a victim, and the associated penalty that the rescuer fails to rescue the victim are taken into consideration, and are transformed into a goal function which reflects the trade-off between the total successful rescue costs and the failure penalties; the RNN is utilised as a fast optimisation algorithm to minimise the goal function with a gradient descent learning procedure; in the RNN, each possible rescuer-victim pair is considered as a neuron, and the neuron with the highest excitation probability after the training process is selected as the decision. The study in [19] proposes a task allocation algorithm for heterogeneous agents in hazard environments with respect to time, space and communication restrictions. As aforementioned in section 2.2, the work in [7] employs an ordinary differential equation model to redistribute rescue robots among various tasks in a decentralised manner without inter-robot communications.

\section{Conclusion}

In this paper, we provide a systemic review to challenges and solutions of the emergency search and rescue problem, we first review the history and evolution of this research area, 
followed by the various frameworks of rescue systems along with different pedestrian models used in mimicking the movement patterns of a search or rescue process. After that, we summarise representative algorithms used to rescue victims. The work is a major supplement to our previous work in [14], they each cover one of the two main branches of the studies in emergency management.

\section{References}

[1] Gelenbe E, Han Q. Near-Optimal Emergency Evacuation with Rescuer Allocation. In: In Proceedings of the 4th International Workshop on Pervasive Networks for Emergency Management (PerNEM'14). IEEE; 2014. p. 1-6.

[2] Orefice M, Pirinoli P, Dassano G. Electrically-small wearable antennas for emergency services applications. In: Antenna Technology (iWAT), 2016 International Workshop on. IEEE; 2016. p. 131-4.

[3] Li S, Zhan A, Wu X, Chen G. ERN: Emergence rescue navigation with wireless sensor networks. In: Parallel and Distributed Systems (ICPADS), 2009 15th International Conference on. IEEE; 2009. p. 361-8.

[4] Wu W, Cao J, Zheng Y, Zheng YP. WAITER: A Wearable Personal Healthcare and Emergency Aid System. In: 2008 Sixth Annual IEEE International Conference on Pervasive Computing and Communications (PerCom); 2008. p. 680-5.

[5] Bozkurt A, Roberts DL, Sherman BL, Brugarolas R, Mealin S, Majikes J, et al. Toward cyber-enhanced working dogs for search and rescue. IEEE Intelligent Systems. 2014;29(6):32-9.

[6] Halász A, Hsieh MA, Berman S, Kumar V. Dynamic redistribution of a swarm of robots among multiple sites. In: Intelligent Robots and Systems, 2007. IROS 2007. IEEE/RSJ International Conference on. IEEE; 2007. p. 2320-5.

[7] Berman S, Halász Á, Hsieh MA, Kumar V. Optimized stochastic policies for task allocation in swarms of robots. IEEE Transactions on Robotics. 2009;25(4):927-37.

[8] Iwano Y, Osuka K, Amano H. Proposal of a Rescue Robot System in Nuclear-Power Plants -Rescue Activity via Small Vehicle Robots -. In: Robotics and Biomimetics, 2004. ROBIO 2004. IEEE International Conference on; 2004. p. 227-32.

[9] Ventura R, Lima PU. Search and rescue robots: The civil protection teams of the future. In: Emerging Security Technologies (EST), 2012 Third International Conference on. IEEE; 2012. p. 12-9.

[10] Jacoff A, Messina E, Weiss B, Tadokoro S, Nakagawa Y, et al. Test arenas and performance metrics for urban search and rescue robots. In: Intelligent Robots and Systems, 2003.(IROS 2003). Proceedings. 2003 IEEE/RSJ International Conference on. vol. 4. IEEE; 2003. p. 3396-403.

[11] Ventura R, Lima PU. Search and Rescue Robots: The Civil Protection Teams of the Future. In: Emerging Security Technologies (EST), 2012 Third International Conference on; 2012. p. 12-9.

[12] Gautam A, Mohan S. A review of research in multi-robot systems. In: Industrial and Information Systems (ICIIS), 2012 7th IEEE International Conference on. IEEE; 2012. p. 1-5.

[13] Gelenbe E, Cao Y. Autonomous search for mines. European Journal of Operational Research. 1998;108(2):319-33.

[14] Bi H, Gelenbe E. A survey of algorithms and systems for evacuating people in confined spaces. Electronics. 2019;8(6):711.

[15] Pugh J, Martinoli A. Inspiring and Modeling Multi-Robot Search with Particle Swarm Optimization. In: Swarm Intelligence Symposium, 2007. SIS 2007. IEEE; 2007. p. 332-9.

[16] Zhu Q, Liang A, Guan H. A PSO-inspired multi-robot search algorithm independent of global information. In: Swarm Intelligence (SIS), 2011 IEEE Symposium on. IEEE; 2011. p. 1-7.

[17] Sutantyo DK, Kernbach S, Levi P, Nepomnyashchikh V, et al. Multi-robot searching algorithm using lévy flight and artificial potential field. In: Safety Security and Rescue Robotics (SSRR), 2010 IEEE International Workshop on. IEEE; 2010. p. 1-6.

[18] Gelenbe E, Timotheou S. Random neural networks with synchronized interactions. Neural Computation. 2008;20(9):2308-24.

[19] Su X, Zhang M, Bai Q. Dynamic task allocation for heterogeneous agents in disaster environments under time, space and communication constraints. The Computer Journal. 2015;58(8):1776-91. 